\title{
The Impact of Pregnancy on Hemorrhagic Stroke in Young Women
}

\author{
Eliza C. Miller ${ }^{a}$ Kathryn M. Sundheim ${ }^{a} \quad$ Joshua Z. Willey ${ }^{a} \quad$ Amelia K. Boehme $^{a}$ \\ Dritan Agalliu $^{\mathrm{a}-\mathrm{c}}$ Randolph S. Marshalla \\ ${ }^{a}$ Department of Neurology, New York, NY, USA; ${ }^{b}$ Departments of Pathology and Cell Biology and Pharmacology, New \\ York, NY, USA; 'C Columbia Neuroscience Translational Initiative Center, Vagelos College of Physicians and Surgeons, \\ Columbia University Irving Medical Center, New York, NY, USA
}

\section{Keywords}

Hemorrhagic stroke · Stroke etiology · Stroke in pregnancy

\begin{abstract}
Background: Pregnancy is a sex-specific risk factor for causing hemorrhagic stroke (HS) in young adults. Unique physiological characteristics during pregnancy may alter the relative risk for HS in pregnant/postpartum (PP) women compared to HS in other young women. We compared patient characteristics and HS subtypes between young nonpregnant and PP women. Methods: We reviewed the medical records of all women 18-45 years old admitted to our center with HS from October 15, 2008 through March 31, 2015, and compared patient characteristics and stroke mechanisms using logistic regression. Results: Of the 130 young women with HS during the study period, 111 were non-PP women, and 19 PP women. PP women had lower proportions of vascular risk factors such as hypertension, prior stroke, and smoking, and a higher proportion of migraine (36.8 vs. $14.4 \%, p=0.01$ ). After adjusting for hypertension, smoking, migraine, prior stroke and prior myocardial infarction, PP women had lower odds of having an underlying vascular lesion (OR $0.14,95 \% \mathrm{Cl} 0.04-0.44, p=$
\end{abstract}

0.0009) and a higher proportion of the reversible cerebral vasoconstriction syndrome (RCVS) as cause of their HS. Conclusions: Women with pregnancy-associated HS had fewer cerebrovascular risk factors, lower odds of having underlying vascular lesions, and higher proportion of migraine and RCVS compared with similar-aged nonpregnant women. Pregnancy-associated HS appears to represent a unique pathophysiological process, requiring targeted study.

(c) 2018 S. Karger AG, Basel

\section{Introduction}

Hemorrhagic stroke (HS) accounts for one-third to one-half of strokes in young adults between 20 and 44 years of age $[1,2]$. Among sex-specific risk factors for HS in the young, pregnancy plays a prominent role $[3,4]$. Prior research has suggested that pregnancy-associated HS may differ significantly in pathophysiology when compared with HS in other young adults [4], but epidemiological studies of this disorder [5] lack many clinical details that may help to better elucidate the underlying pathophysiology.

\section{KARGER}

(c) 2018 S. Karger AG, Basel

E-Mail karger@karger.com

www.karger.com/ced
Eliza C. Miller, MD

The Neurological Institute of New York

710 W. 168th St., 6th floor

New York, NY 10032 (USA)

E-Mailecm2137@cumc.columbia.edu 


\section{Aims}

We compared causes of HS and comorbidities in pregnant/postpartum (PP) young women with HS in other young women admitted to our center, hypothesizing that the subtypes of HS would differ between groups, and thus suggest differences in underlying pathophysiology.

\section{Methods}

Permission to review the electronic medical records for all patients ranging in age from 18-45 years old with HS from January 1, 2008 through March 15, 2015 was granted by the Institutional Review Board of Columbia University Medical Center, and the requirement for informed consent was waived. All women aged 1845 at the time of the index event admitted or diagnosed during admission with HS, specifically intracerebral hemorrhage (ICH) or subarachnoid hemorrhage (SAH), were included in the cohort. Women with traumatic $\mathrm{ICH}$, traumatic $\mathrm{SAH}$, or hemorrhagic conversion of an ischemic stroke were excluded from the analysis. Those with ICH and SAH due to venous infarction with hemorrhage from cerebral venous sinus or cortical vein thrombosis were also excluded; while pregnancy is a known risk factor for venous sinus or cortical vein thrombosis, and can cause hemorrhage, we chose to exclude these cases as the underlying pathophysiology is thrombotic, not hemorrhagic. Of 6,842 total stroke patients included in the database during the study period, 130 met criteria for inclusion into the study.

Participants were considered to have pregnancy-associated HS if the index event occurred during pregnancy or up to 12 weeks postpartum [6]. We reviewed participants' electronic medical records for comorbidities and stroke mechanisms. We categorized HS into 6 mechanisms: primary ICH (defined as ICH with no underlying vascular lesion and without presence of the reversible cerebral vasoconstriction syndrome [RCVS]); ICH with underlying vascular malformation (arteriovenous malformation or cavernous malformation); ICH due to RCVS, aneurysmal SAH, non-aneurysmal SAH due to RCVS, or other SAH (such as perimesencephalic SAH). RCVS was defined as single or recurrent thunderclap headache with concomitant ICH or cortical SAH and absence of an underlying vascular lesion, in accordance with criteria shown to have $>96 \%$ specificity for this disorder [7]. Patients were considered to have an underlying vascular lesion if the presence of the lesion was confirmed on cerebral vessel imaging (computed tomographic angiography [CTA], digital subtraction angiography [DSA], or magnetic resonance angiography [MRA]).

\section{Statistical Analysis}

Groups were compared using chi-squared tests for categorical variables, Fisher's exact test where appropriate, and Wilcoxon rank sum test or Kruskal-Wallis test for continuous variables. Crude and adjusted logistic regression analyses were used to assess odds of having a vascular lesion between non-PP women and PP women. All analyses were performed using SAS 9.3 (SAS Institute Inc., Cary, NC, USA).

HS and pregnancy

\section{Results}

There were 130 young women admitted with HS during the study period: 111 (85.4\%) non-PP women and 19 (14.6\%) PP women. All 19 of the PP women had dedicated cerebrovascular imaging (either CTA, MRA, DSA or a combination of these). Of the 111 non-PP women, 106 (95.5\%) had dedicated cerebrovascular imaging; of the 5 women who did not, 3 had magnetic resonance imaging (MRI) of the brain with and without intravenous gadolinium contrast, 1 had MRI of the brain without contrast, and 1 had only CT of the brain without contrast due to a massive pontine hemorrhage resulting in rapid clinical deterioration. Baseline characteristics and imaging modalities used for the 2 groups are shown in Table 1. There were striking differences in stroke subtypes between groups as presented in Figure 1. Compared with non-PP women, PP women had fewer vascular risk factors, higher proportions of migraine, lower proportions of aneurysmal SAH, and higher proportions of RCVS as a cause for HS (13 of 19 PP women [68.4\%] vs. 2 of 130 non-PP women [1.5\%], $p<0.0001$ ). $\mathrm{PP}$ women had lower odds of having an underlying vascular lesion (OR 0.14, 95\% CI 0.04-0.44, $p=0.0009$ ) when compared with non-PP women, after adjusting for hypertension, smoking, migraine, prior stroke, or prior myocardial infarction. Case characteristics in the PP group are detailed in Table 2. The timing of stroke with regard to subtype was also striking. Among PP women, HS clustered in the second trimester, all of which were due to a vascular lesion, and in the second week postpartum, none of which were due to a vascular lesion (Fig. 2). Of the 12 postpartum HS, 10 (83.3\%) were due to RCVS. Among the 19 PP women, 6 (31.6\%) had preeclampsia during pregnancy.

\section{Discussion}

The distribution of etiologies and comorbidities for HS in young women differs by pregnancy/postpartum status. In our cohort of 130 women aged 18-45, PP women had lower odds of having underlying vascular lesions, lower proportion of vascular risk factors, and a higher proportion of migraine compared with non-PP women. RCVS, a rare cause of HS in non-PP women, accounted for two-thirds of pregnancy-associated HS and almost all of the strokes in the postpartum period.

Our study adds to a growing body of evidence suggesting that the pregnancy-associated stroke, and in particu- 
Table 1. Baseline characteristics for women aged 18-45 with hemorrhagic stroke

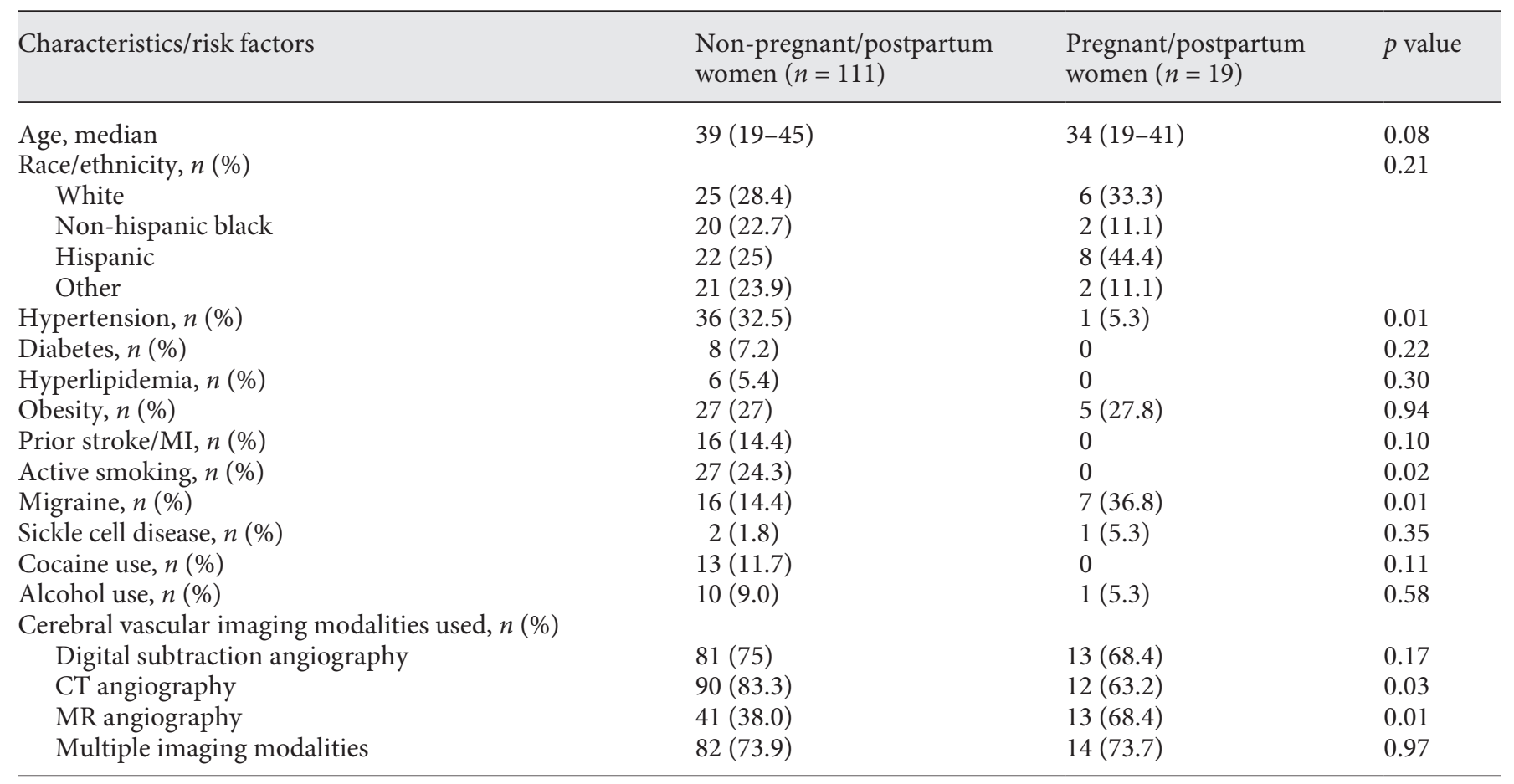

MI, myocardial infarction; CT, computed tomography; MR, magnetic resonance.

lar the postpartum stroke, represents a unique pathophysiological entity.

Almost one-third of the PP women in our cohort had preeclampsia, which is strongly associated with HS [8]. Preeclampsia, an endotheliopathy characterized by hypertension during pregnancy and multi-organ dysfunction, shares common features with RCVS and the posterior reversible encephalopathy syndrome $[9,10]$. The prominent role that RCVS appears to play in the pathogenesis of postpartum stroke suggests that RCVS may represent a spectrum of pregnancy-associated angiopathy or vasculitis [11]. Postpartum angiopathy has been recognized, since Call and Fleming described it in 1988 [12], and like other forms of RCVS, can range in severity from mild to fulminant [13-15].

"Pregnancy-related angiopathy" may be a more apt term for this phenomenon, which may have features not present in other forms of RCVS. For example, animal studies have shown that exposing healthy rat brains to proinflammatory cytokines present in sera from normal pregnant rats led to activation of microglia and neuronal hyperexcitability. These effects were primarily mediated by tumor necrosis factor-alpha and were absent when the blood-brain barrier (BBB) was intact, thereby suggesting that impairment in BBB function may partially underlie the increased vulnerability for PRES and pregnancy-related stroke [16]. In support of this hypothesis, follow-up in vitro studies demonstrated that serum from healthy pregnant women increased BBB permeability of rat cerebral arterioles by sixfold compared to controls; moreover, serum from preeclamptic women had an even larger effect size (18-fold) than serum from healthy pregnant women in increasing BBB permeability [17]. Placental ischemia, a key disturbance in preeclampsia, has been shown to impair cerebral blood flow autoregulation and enhance BBB permeability in rats, an effect that is mediated primarily by tumor necrosis factor-alpha $[18,19]$. Additional studies demonstrated that reduced uterine perfusion pressure in pregnant rats led to increased vascular permeability and PRES-like changes [20]. Preeclampsia may represent the extreme on a continuum of pregnancy-induced cerebrovascular changes induced by either proinflammatory or hypertension-related factors that impair endothelial function. Animal models may be critical to enhance our understanding of the mechanisms that lead to pregnancy-associated stroke.

Headache is often a feature of preeclampsia, and by diagnostic criteria established in 2013, is sufficient to
12

Cerebrovasc Dis 2018;46:10-15 DOI: $10.1159 / 000490803$
Miller/Sundheim/Willey/Boehme/ Agalliu/Marshall 
Table 2. Characteristics, stroke mechanisms, and outcomes of PP women with hemorrhagic stroke $(n=19)$

\begin{tabular}{|c|c|c|c|c|c|c|c|}
\hline Age & Parity & Medical comorbidities & Pregnancy complications & Stroke type & $\begin{array}{l}\text { Stroke } \\
\text { mechanism }\end{array}$ & $\begin{array}{l}\text { Stroke } \\
\text { timing }\end{array}$ & $\begin{array}{l}\text { Discharge } \\
\text { mRS }\end{array}$ \\
\hline 19 & G2P1 & None & Intrauterine fetal demise after stroke & $\mathrm{ICH}$ & AVM & 19 weeks & 5 \\
\hline 19 & G1P0 & Migraine, pneumonia & Preeclampsia & SAH & RCVS & 33 weeks & 0 \\
\hline 21 & $\mathrm{G} 2 \mathrm{P} 2$ & Sickle cell disease & Chorioamnionitis, preeclampsia & $\mathrm{ICH} / \mathrm{SAH}$ & RCVS & PP day 11 & 4 \\
\hline 29 & $\mathrm{G} 2 \mathrm{P} 2$ & Obesity & None & $\mathrm{ICH}$ & AVM & 27 weeks & 0 \\
\hline 30 & G1P1 & None & None & SAH & Aneurysm & 17 weeks & 2 \\
\hline 30 & G5P5 & Migraine & None & $\mathrm{ICH}$ & RCVS & PP day 6 & 1 \\
\hline 32 & G1P1 & Obesity, chronic hypertension & Preeclampsia & $\mathrm{ICH}$ & Hypertensive & PP day 1 & 4 \\
\hline 32 & G4P4 & Migraine & Preeclampsia & $\mathrm{SAH}$ & RCVS & PP day 9 & 0 \\
\hline 33 & $\mathrm{G} 2 \mathrm{P} 1$ & Migraine, obesity & None & $\mathrm{SAH}$ & RCVS & PP day 8 & 0 \\
\hline 34 & G1P1 & UTI & Gestational diabetes & $\mathrm{ICH}$ & AVM & 22 weeks & 4 \\
\hline 35 & $\mathrm{G} 2 \mathrm{P} 2$ & UTI, asthma & None & $\mathrm{ICH}$ & AVM & 24 weeks & 4 \\
\hline 35 & G1P1 & None & Elective termination & $\mathrm{ICH}$ & AVM & 15 weeks & 5 \\
\hline 37 & G1P1 & Polycystic ovarian syndrome & Cholestasis of pregnancy & SAH & RCVS & PP day 1 & 0 \\
\hline 37 & G4P4 & None & Preeclampsia & SAH & RCVS & PP day 8 & 0 \\
\hline 37 & G12P13 & Migraine & Multiple gestation & $\mathrm{SAH}$ & RCVS & PP day 12 & 1 \\
\hline 38 & G1P2 & Obesity, pseudotumor cerebri & Multiple gestation & $\mathrm{SAH}$ & RCVS & PP day 11 & 1 \\
\hline 38 & G3P3 & Migraine & None & $\mathrm{ICH} / \mathrm{SAH}$ & RCVS & PP day 10 & 4 \\
\hline 40 & G1P1 & Migraine, obesity, Hashimoto thyroiditis & None & $\mathrm{SAH}$ & RCVS & PP day 7 & 0 \\
\hline 41 & G1P1 & None & Preeclampsia & $\mathrm{ICH}$ & Hypertensive & PP day 21 & 3 \\
\hline
\end{tabular}

mRS, modified Rankin scale score; G, gravida; P, para; ICH, intracerebral hemorrhage; SAH, subarachnoid hemorrhage; AVM, arteriovenous malformation; RCVS, reversible cerebral vasoconstriction syndrome; UTI, urinary tract infection; PP, pregnant/post partum.

Fig. 1. Hemorrhagic stroke subtypes in women aged 18-45. Cumulative bar graph of hemorrhagic stroke subtypes in nonpregnant women $(n=111)$ and pregnant/ postpartum women $(n=19)$. Some patients had more than one subtype. * Primary ICH was defined as ICH with no underlying vascular lesion. ICH, intracerebral hemorrhage; SAH, subarachnoid hemorrhage; RCVS, reversible cerebral vasoconstriction syndrome.

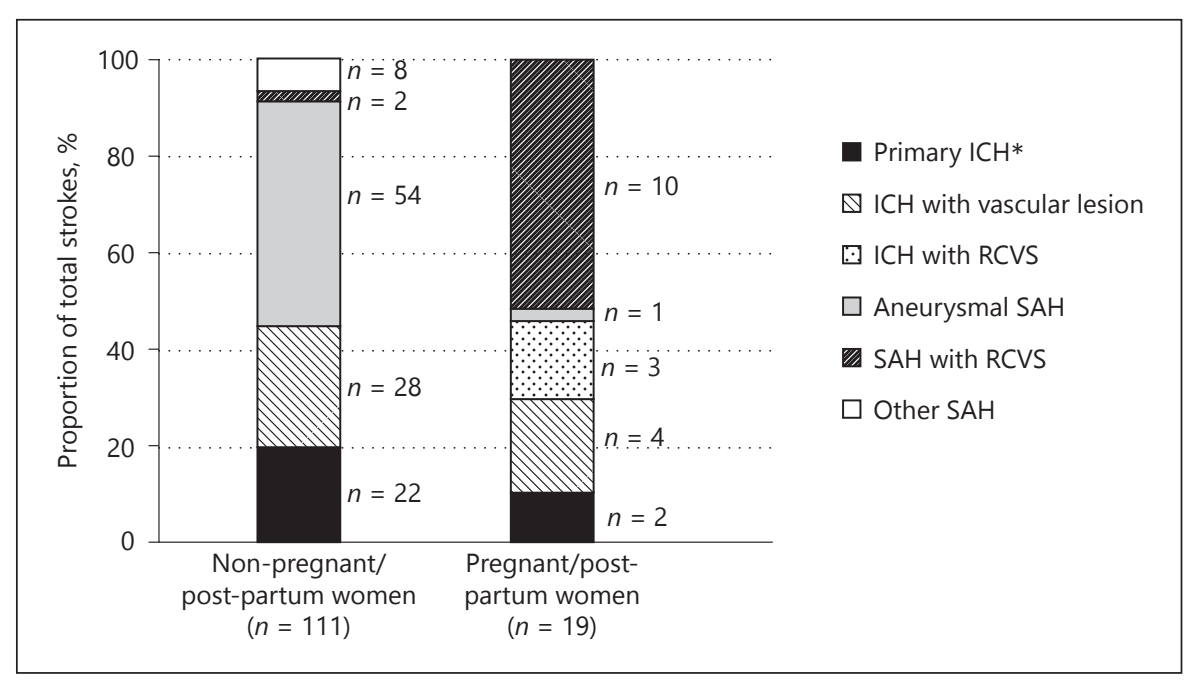

qualify preeclampsia as "with severe features" even in the absence of proteinuria [21, 22]. Migraine has been associated both with hypertensive disorders of pregnancy [23] and hemorrhagic complications of RCVS [24]. The incidence of RCVS in the general population is unknown, but the condition is likely underdiagnosed where some patients with self-reported "migraines" may actually have RCVS [25-27]. In our cohort, the history of migraines appeared more frequently with HS in PP women, but not in other young women. Thus, it is possible that migraine may represent either a risk factor or a marker for increased risk of pregnancy-associated HS in some women. The relationship between migraine, RCVS, preeclampsia, and pregnancy-related stroke is complex and not well characterized, and should be explored in future studies.

The second-trimester cluster of hemorrhages due to vascular lesions is also intriguing. Whether pregnancy in- 
Fig. 2. Timing of pregnancy-associated hemorrhagic strokes in women 18-45 years old at a tertiary care center, 20082015. Light gray circles indicate SAH. Dark gray circles indicate ICH. Red perimeter indicates concomitant RCVS. Doughnut indicates pregnancy complicated by PEC. No strokes occurred in the first trimester or after postpartum day 21. All the strokes in the second trimester were due to the rupture of vascular lesions ( 1 aneurysm, 5 arteriovenous malformations). PEC, preeclampsia; SAH, subarachnoid hemorrhage; ICH, intracerebral hemorrhage; RCVS, reversible cerebral vasoconstriction syndrome.

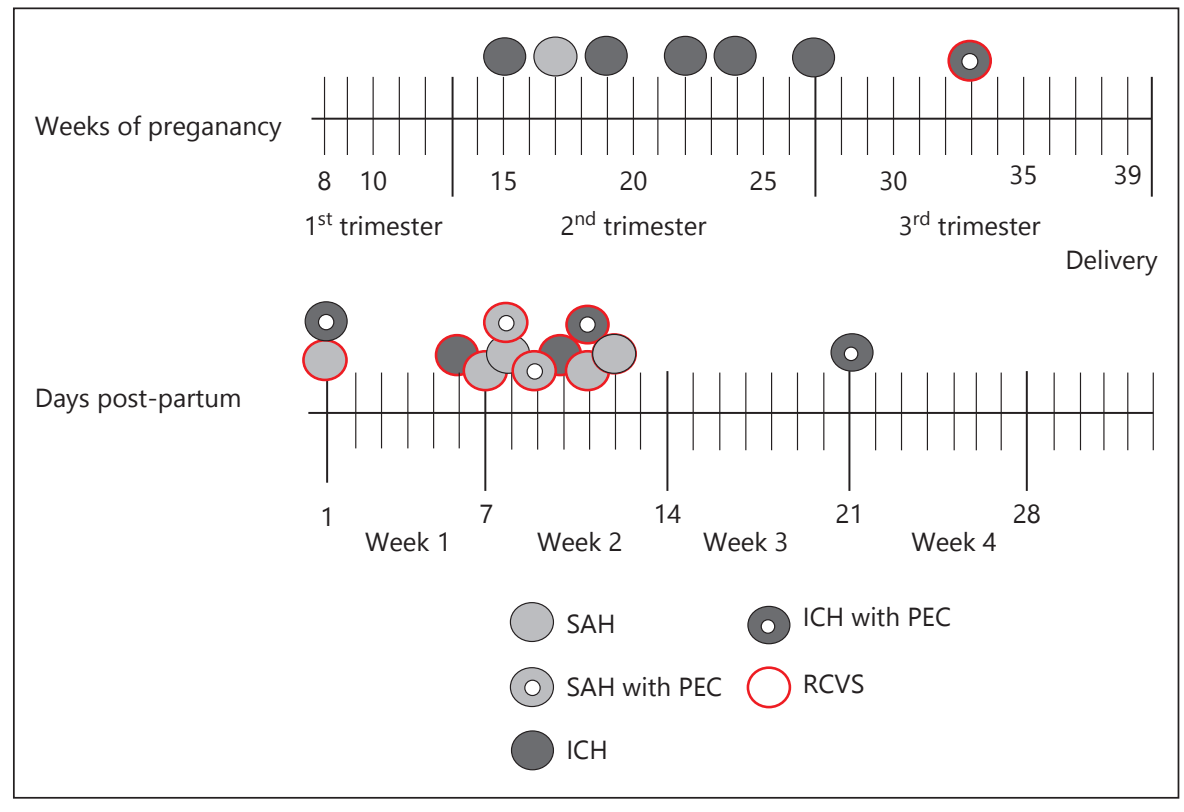

creases the risk of bleeding from unruptured vascular lesions is debated, with some data indicating increased risk $[28,29]$, and other data not supporting this [30-32]. However, previous series have also shown a tendency for ruptures, when they do happen, to occur in the second or the early third trimester $[28,31]$. This could be related to the dramatic increase in plasma volume seen in the second trimester, reaching its peak at 32 weeks gestation, along with hormonally mediated alterations in vesselwall elasticity and compliance that also peak during the second trimester [33]. This also warrants further investigation, as it may have implications for management of unruptured lesions.

Our exploratory study has limitations. It is a small sample confined to a single tertiary care center, which may limit the generalizability of our results. While all the PP women had vessel imaging, lack of vessel imaging in a few non-PP women may have led to under-diagnosis of vascular lesions in that group. However, since under-diagnosis would tend to bias towards a smaller difference between groups, this is unlikely to have changed our results.

\section{Conclusions}

In our sample of young women with HS, women who had pregnancy-related HS had fewer cerebrovascular risk factors and more migraine, were less likely to have underlying vascular lesions, and had higher proportions of
RCVS as the cause of their stroke than similarly aged nonPP women. Pregnancy-associated HS may be caused by a unique pregnancy-related angiopathy, producing a specific pathophysiology, and requiring a targeted study.

\section{Disclosure Statement}

The authors declare that there are no conflicts of interest to disclose.

\section{Funding Source}

NIH/NINDS 5U10NS086728-05 StrokeNet Training Core (E.C.M.); NINDS 1 U10 NS086728 (R.S.M.); NIH/NHLBI R01 HL116995-01 (D.A.); NIMH R56 MH109987-01A1 (D.A.), the Leducq Foundation (D.A.), an unrestricted gift from John. F. Castle (D.A. and R.S.M.).

\section{References}

Miller/Sundheim/Willey/Boehme/ Agalliu/Marshall
1 Kissela BM, Khoury JC, Alwell K, Moomaw CJ, Woo D, Adeoye O, et al: Age at stroke: temporal trends in stroke incidence in a large, biracial population. Neurology 2012;79: 1781-1787.

2 Jacobs BS, Boden-Albala B, Lin IF, Sacco RL: Stroke in the young in the Northern Manhattan stroke study. Stroke 2002;33:2789-2793.

3 Miller EC, Gatollari HJ, Too G, Boehme AK, Leffert L, Elkind MS, et al: Risk of pregnancyassociated stroke across age groups in New York state. JAMA Neurol 2016;73:14611467. 
4 Leffert LR, Clancy CR, Bateman BT, Cox M, Schulte PJ, Smith EE, et al: Patient characteristics and outcomes after hemorrhagic stroke in pregnancy. Circ Cardiovasc Qual Outcomes 2015;8(6 suppl 3):S170-S178.

5 Swartz RH, Cayley ML, Foley N, Ladhani NNN, Leffert L, Bushnell C, et al: The incidence of pregnancy-related stroke: a systematic review and meta-analysis. Int J Stroke 2017;12:687-697.

6 Kamel H, Navi BB, Sriram N, Hovsepian DA, Devereux RB, Elkind MS: Risk of a thrombotic event after the 6-week postpartum period. N Engl J Med 2014;370:1307-1315.

7 Singhal AB, Topcuoglu MA, Fok JW, Kursun O, Nogueira RG, Frosch MP, et al: Reversible cerebral vasoconstriction syndromes and primary angiitis of the central nervous system: clinical, imaging, and angiographic comparison. Ann Neurol 2016;79:882-894.

8 Crovetto F, Somigliana E, Peguero A, Figueras F: Stroke during pregnancy and pre-eclampsia. Curr Opin Obstet Gynecol 2013;25 425-432.

9 Hammer ES, Cipolla MJ: Cerebrovascular Dysfunction in Preeclamptic Pregnancies. Curr Hypertens Rep 2015;17:64.

10 Bembalgi S, Kamate V, Shruthi KR: A study of eclampsia cases associated with posterior reversible encephalopathy syndrome. J Clin Diagn Res 2015;9:QC05-OC07.

11 Razmara A, Bakhadirov K, Batra A, Feske SK: Cerebrovascular complications of pregnancy and the postpartum period. Curr Cardiol Rep 2014; 16:532.

12 Call GK, Fleming MC, Sealfon S, Levine H, Kistler JP, Fisher CM: Reversible cerebral segmental vasoconstriction. Stroke 1988;19: 1159-1170.

13 Ursell MR, Marras CL, Farb R, Rowed DW, Black SE, Perry JR: Recurrent intracranial hemorrhage due to postpartum cerebral angiopathy: implications for management. Stroke 1998;29:1995-1998.

14 Wong SH, Dougan C, Chatterjee K, Fletcher NA, White RP: Recurrent thunderclap headaches and multilobar intracerebral haemor- rhages: two cases of reversible cerebral vasoconstriction syndrome (RCVS). Cephalalgia 2009;29:791-795.

15 Fugate JE, Wijdicks EFM, Parisi JE, Kallmes DF, Cloft HJ, Flemming KD, et al: Fulminant postpartum cerebral vasoconstriction syndrome. Arch Neurol 2012;69:111-117.

16 Cipolla MJ, Pusic AD, Grinberg YY, Chapman AC, Poynter ME, Kraig RP: Pregnant serum induces neuroinflammation and seizure activity via TNFa. Exp Neurol 2012;234:398-404.

17 Amburgey OA, Chapman AC, May V, Bernstein IM, Cipolla MJ: Plasma from preeclamptic women increases blood-brain barrier permeability: role of vascular endothelial growth factor signaling. Hypertension 2010;56:10031008.

18 Warrington JP, Fan F, Murphy SR, Roman RJ, Drummond HA, Granger JP, et al: Placental ischemia in pregnant rats impairs cerebral blood flow autoregulation and increases blood-brain barrier permeability. Physiol Rep 2014;2.pii:e12134.

19 Warrington JP, Drummond HA, Granger JP, Ryan MJ: Placental ischemia-induced increases in brain water content and cerebrovascular permeability: role of TNF- $\alpha$. Am J Physiol Regul Integr Comp Physiol 2015; 309:R1425-R1431.

20 Porcello Marrone LC, Gadonski G, de Oliveira Laguna G, Poli-de-Figueiredo CE, Pinheiro da Costa BE, Lopes MFT, et al: Blood-brain barrier breakdown in reduced uterine perfusion pressure: a possible model of posterior reversible encephalopathy syndrome. J Stroke Cerebrovasc Dis 2014;23:2075-2079.

21 American College of Obstetricians and Gynecologists, Task Force on Hypertension in Pregnancy: Hypertension in pregnancy. Report of the American College of Obstetricians and Gynecologists' Task Force on Hypertension in Pregnancy, 2013, pp 1122-1131.

22 Mol BWJ, Roberts CT, Thangaratinam S, Magee LA, et al: Pre-eclampsia. Lancet 2016;387: 999-1011.

23 Wabnitz A, Bushnell C: Migraine, cardiovascular disease, and stroke during pregnancy: systematic review of the literature. Cephalalgia 2015;35:132-139.

24 Ducros A, Fiedler U, Porcher R, Boukobza M, Stapf C, Bousser MG: Hemorrhagic manifestations of reversible cerebral vasoconstriction syndrome: frequency, features, and risk factors. Stroke 2010;41:2505-2511.

25 Singhal AB, Hajj-Ali RA, Topcuoglu MA, Fok $J$, Bena J, Yang D, et al: Reversible cerebral vasoconstriction syndromes: analysis of 139 cases. Arch Neurol 2011;68:1005-1012.

26 Ducros A: Reversible cerebral vasoconstriction syndrome. Lancet Neurol 2012;11:906917.

27 Chen SP, Fuh JL, Lirng JF, Wang YF, Wang SJ: Recurrence of reversible cerebral vasoconstriction syndrome: a long-term follow-up study. Neurology 2015;84:1552-1558.

28 Porras JL, Yang W, Philadelphia E, Law J, Garzon-Muvdi T, Caplan JM, et al: Hemorrhage risk of brain arteriovenous malformations during pregnancy and puerperium in a North American cohort. Stroke 2017;48: 1507-1513.

29 Gross BA, Du R: Hemorrhage from arteriovenous malformations during pregnancy. Neurosurgery 2012;71:349-355

30 Dias MS, Sekhar LN: Intracranial hemorrhage from aneurysms and arteriovenous malformations during pregnancy and the puerperium. Neurosurgery 1990;27:855-865.

31 Liu XJ, Wang S, Zhao YL, Teo M, Guo P, Zhang D, et al: Risk of cerebral arteriovenous malformation rupture during pregnancy and puerperium. Neurology 2014;82:1798-1803.

32 Horton JC, Chambers WA, Lyons SL, Adams RD, Kjellberg RN: Pregnancy and the risk of hemorrhage from cerebral arteriovenous malformations. Neurosurgery 1990;27:867871.

33 Monga M, Mastrobattista JM: Maternal Cardiovascular, Respiratory, and Renal Adaptation to Pregnancy; in Creasy RK, Resnik R, Iams JD, Lockwood C, Moore T, Greene M (eds): Creasy and Resnik's Maternal Fetal Medicine, ed 7. Philadelphia, PA, 2014, pp 93-99.e3. 